\title{
miR-29a-3p regulates the epithelial-mesenchymal transition via the SPARC/ERK signaling pathway in human bronchial epithelial cells
}

\author{
XIAOBO ZHANG, JUN XIE, HONGMEI SUN, QIN WEI and GUANGMIN NONG \\ Pediatric Department, First Affiliated Hospital of Guangxi Medical University, \\ Nanning, Guangxi Zhuang Autonomous Region 530021, P.R. China
}

Received November 29, 2020; Accepted May 24, 2021

DOI: $10.3892 /$ ijmm.2021.5004

\begin{abstract}
Neutrophilic asthma (NA) is a subtype of asthma that responds poorly to corticosteroid treatment. In certain diseases, microRNA (miR)-29a-3p is considered to be a key regulatory molecule for remodeling of the extracellular matrix. However, the effect of miR-29a-3p on airway remodeling is unknown. The present study aimed to investigate the role of miR-29a-3p in NA. A mouse model of NA was established and these animals were compared to normal controls. Both groups of mice were subjected to lung function tests and histopathological analysis. Human bronchial epithelial cells (16HBE) were grown in culture and incubated with secreted protein acidic rich in cysteine (SPARC) and a miR-29a-3p mimic. The expression of miR-29a-3p, SPARC and epithelial-mesenchymal transition (EMT)-related markers were measured using reverse transcription-quantitative PCR and western blotting. Luciferase reporter assay was performed to identify the direct regulatory relationship between miR-29a-3p and SPARC. miR-29a-3p expression was significantly decreased, while SPARC expression was increased in the NA mouse model with a phenotype of EMT. Overexpression of SPARC downregulated the expression of E-cadherin, while it increased the expression of vimentin in 16HBE cells. miR-29a-3p administration reversed the SPARC-induced effects on E-cadherin and vimentin expression. Luciferase assays confirmed that SPARC was the target gene for miR-29a-3p. Furthermore, SPARC overexpression increased the protein expression of phosphorylated (p)-ERK, while transfection with miR-29a-3p mimics significantly inhibited this increase. The data suggested that EMT in
\end{abstract}

Correspondence to: Professor Guangmin Nong, Pediatric Department, First Affiliated Hospital of Guangxi Medical University, 6 Shuangyong Road, Nanning, Guangxi Zhuang Autonomous Region 530021, P.R. China

E-mail: ngmprof8525@126.com

Key words: microRNA-29a-3p, secreted protein acidic rich in cysteine, epithelial-mesenchymal transition, bronchial epithelial cell, neutrophilic asthma the NA mouse model was associated with decreased levels of miR-29a-3p and elevated SPARC. Furthermore, SPARC could induce the formation of EMT in $16 \mathrm{HBE}$ cells in vitro and this was directly targeted by miR-29a-3p and mediated by $p$-ERK, suggesting that miR-29a-3p may participate in the airway remodeling of NA.

\section{Introduction}

Asthma is the most common chronic childhood disease (1). Neutrophilic asthma (NA) is a subtype of asthma, which occurs in $15-25 \%$ of cases and responds poorly to glucocorticoid treatment (2). NA is characterized by neutrophil-mediated inflammation of the airway, and can result in reduced bronchodilator reversibility and fixed airflow obstruction, which are also the features of chronic obstructive pulmonary disease $(3,4)$. Understanding the immunopathology of NA may result in the discovery of targeted treatments for patients (3).

MicroRNAs (miRNAs/miRs) are small non-coding RNAs that regulate the post-transcriptional expression of multiple genes by complementary binding to the 3 ' untranslated region (3'UTR) of the target gene (5). miR-29a-3p has been demonstrated to participate in a variety of diseases and different pathological processes, including cell proliferation, apoptosis, fibrosis and immunomodulation (6,7). Epithelial-mesenchymal transition (EMT) is a process in which epithelial cells lose the epithelial phenotype and undergo the transition to typical mesenchymal characteristics. Recent evidence has suggested that EMT is involved in airway remodeling and the development of asthma $(8,9)$. More importantly, miR-29a-3p has been implicated in the inhibition of gene expression in EMT and metastasis (6). However, the effect of miR-29a-3p on EMT in NA remains unknown.

Thus, the present study aimed to investigate the role of miR-29a-3p in NA. To study the role of miR-29a-3p in NA, a mouse model of NA was established and these animals were compared to normal controls. Both groups of mice were subjected to lung function tests and histopathological analysis to confirm the induction of the NA model. Human bronchial epithelial cells (16HBE) were grown in culture, and in vitro experiments were performed. The expression levels of miR-29a-3p, secreted protein acidic rich in cysteine (SPARC) 
and EMT-related markers were measured and luciferase reporter assays were performed to identify the direct regulatory relationship between miR-29a-3p and SPARC.

\section{Materials and methods}

Animals. Female C57BL/6 mice ( $\mathrm{n}=12$; certificate no. SCXK 2020-0003) aged 6-8 weeks and weighing 18-20 g were purchased from Guangxi Medical University Animal Center (license no. SYXK 2020-0004). Mice were housed under specific pathogen-free conditions with a relatively stable temperature $\left(20-24^{\circ} \mathrm{C}\right)$ and humidity $(55 \pm 10 \%)$ at 12 -h light/dark cycles. All animal experiments were approved by The Ethics Committee of The First Affiliated Hospital of Guangxi Medical University [approval no. 2019 (KY-E-035); Nanning, China].

Animal groups. Mice were randomly divided into the normal control group (NC) and a NA group (NA) (n=6 in each group). The mouse model with NA was established using previously outlined protocols $(10,11)$. Mice were sensitized by airway delivery of $100 \mu \mathrm{g}$ ovalbumin (OVA; Grade II \& V; Sigma-Aldrich; Merck KGaA) and $1 \mu \mathrm{g}$ lipopolysaccharide (LPS; Sigma-Aldrich; Merck KGaA) in a total volume of $50 \mu 1$ PBS on days 1, 7 and 14. The mixture was instilled along the posterior oropharyngeal wall and inhaled into the airway, followed by a challenge with 1\% OVA aerosol for $1 \mathrm{~h}$ from day 21 for 7 consecutive days. The mice in the NC group received the equivalent amount of PBS treatment instead of OVA + LPS for sensitization and challenge.

Lung function measurements. Mice were anesthetized with $1 \%$ pentobarbital sodium ( $50 \mathrm{mg} / \mathrm{kg}$ body weight) by intraperitoneal injection. Measurements of dynamic resistance were assessed using whole-body plethysmography (Buxco ${ }^{\circledR}$ FinePointe Noninvasive Airway Mechanics; Data Sciences International) and induced with methacholine (Sigma-Aldrich; Merck KGaA) at doses of 12.5, 25 and $50 \mathrm{mg} / \mathrm{ml} 24 \mathrm{~h}$ after the final OVA challenge. Each mouse was exposed to aerosolized PBS (baseline) for 3 min followed by the administration of increasing concentrations of methacholine solutions. Airway resistance [enhanced pause (Penh)] values were then evaluated for $5 \mathrm{~min}$. The results were expressed as the percentage of the baseline Penh value for each concentration of methacholine used (12).

Cell classification of bronchoalveolar fluid (BALF). Mice were sacrificed $24 \mathrm{~h}$ after the final aerosolization. Cervical dislocation was used for euthanasia and death was confirmed by the onset of rigor mortis, according to The National Institutes of Health Guide for the Care and Use of Laboratory Animals. The lungs were subjected to bronchoalveolar lavage twice $24 \mathrm{~h}$ after the final aerosolization with $0.5 \mathrm{ml}$ PBS (recovery rate $\geq 80 \%$ ) and the total volume of BALF was $0.8 \mathrm{ml}$. Total and differential cell counts in BALF were determined by Diff-Quick staining (Beijing Solarbio Science \& Technology Co., Ltd.) for $1 \mathrm{~min}$ at room temperature, according to previously outlined protocols (11).

Histopathological analysis. Lungs were fixed in 4\% paraformaldehyde solution for $24 \mathrm{~h}$ at room temperature, and then subjected to gradient alcohol dehydration and paraffin-embedding. Then, the lungs were sliced into 5-7- $\mu \mathrm{m}$ thick sections and subsequently stained with hematoxylin and eosin (H\&E) at room temperature for 2-3 min. An optical microscope (Olympus Corporation) was used to evaluate the general inflammation using previously outlined protocols (11).

Cell culture and transfection. Human bronchial epithelial cells (16HBE) were purchased from FuHeng Biology (cat. no. FH1013) and maintained in Keratinocyte medium (cat. no. 2101; ScienCell Research Laboratories, Inc.) in a humidified atmosphere of $5 \% \mathrm{CO}_{2}$ at $37^{\circ} \mathrm{C}$. Cells were authenticated using the STR genotype test and passed the mycoplasma testing. Cell passage four was used for this study. For lentiviral transfection, pLV-h-SPARC-CMV-MCS-3FLAG-EF1-ZsGreen-PURO [Lentivirus(LV)-SPARC] andpLV-CMV-MCS-3FLAG-EF1-ZsGreen-T2A-PURO (LV-control; both purchased from Sangon Biotech Co., Ltd.) were transfected into 293T cells (National Collection of Authenticated Cell Cultures; National Science \& Technology Infrastructure), 2nd generation system was used [ratio of the lentiviral plasmid $(10 \mu \mathrm{g})$ :packaging vector $(10 \mu \mathrm{g})$ :envelope $(5 \mu \mathrm{g})]$. For virus collection, the supernatant was collected 48 and $72 \mathrm{~h}$ after transfection of the packaged cells, centrifuged at $2,000 \mathrm{xg}$ for $10 \mathrm{~min}$ to remove cell debris, and ultracentrifuged at $82,700 \mathrm{x}$ g for $120 \mathrm{~min}$, then the pellet was resuspend in the culture medium to determine the titer. miR-29a-3p mimic and miR-29a-3p inhibitor were provided by Sangon Biotech Co., Ltd. 16HBE cells $\left(1 \times 10^{6}\right.$ cells $\left./ \mathrm{ml}\right)$ at $60-80 \%$ confluence were treated with LV-SPARC [at 50 multiplicity of infection (MOI)] for $24 \mathrm{~h}$, and then a miR-29a-3p mimic or miR-29a-3p inhibitor $(100 \mathrm{nM})$ mixed with Lipofectamine ${ }^{\circledR} 6000$ transfection reagent (Invitrogen; Thermo Fisher Scientific, Inc.) was transfected into cells at room temperature for $5 \mathrm{~min}$, and incubated for $24 \mathrm{~h}$. The negative control group ( $\mathrm{NeC}$; untreated cells) was set. miRNAs were labeled with FAM, and transfection efficacy was determined by the expression of immunofluorescence using fluorescence microscopy (Olympus Corporation). The cells were lysed for reverse transcription-quantitative (RT-q)PCR and western blotting. The sequences were as follows: miR-29a-3p mimic, 5'-UAGCACCAUCUGAAAUCG GUUA-3'; miR-29a-3p inhibitor, 5'-UAACCGAUUUCAGAU GGUGCUA-3'; miR-29a-3p mimic control, 5'-UUUGUACUA CACAAAAGUACUG-3'; and miR-29a-3p inhibitor control, 5'-CAGUACUUUUGUGUAGUACAAA-3'.

Luciferase reporter gene assay. The target gene of miR-29a-3p was predicted using the online bioinformatics software, TargetScan3.1 (http://www.targetscan.org/vert_71/). Cells were co-transfected with miR-29a-3p mimic or miR mimic control, and psiCHECK-2-SPARC-3'-UTR-wild-type (WT) plasmid or a psiCHECK-2-SPARC-3'-UTR-mutant (MUT) plasmid (Promega Corporation) using Lipofectamine ${ }^{\circledR} 2000$ (Invitrogen; Thermo Fisher Scientific, Inc.). Luciferase activity was measured $48 \mathrm{~h}$ after transfection using Dual-Luciferase system (Promega Corporation) in comparison with Renilla luciferase activity.

$R T-q P C R$. The lung samples and cultured cells were subjected to RNA extraction using TRIzol (cat. no. 15596-026; Invitrogen; Thermo Fisher Scientific, Inc.). cDNA for RNA 
and miRNA were synthesized using the PrimeScript ${ }^{\mathrm{TM}}$ RT Reagent kit with gDNA Eraser (cat. no. RR047A; Takara Bio, Inc.) and Mir- $\mathrm{X}^{\mathrm{TM}}$ miRNA First-Strand Synthesis kit (cat. no. 638313; Takara Bio, Inc.) according to the manufacturer's protocol, respectively. qPCR was performed using an ABI 7500 Real-Time PCR instrument (Applied Biosystems; Thermo Fisher Scientific, Inc.) with TB Green ${ }^{\circledR}$ Premix Ex Taq $^{\text {TM }}$ II (Tli RNase H Plus) (cat. no. RR820A; Takara Bio, Inc.) using the following primers: U6 forward, 5'-GCTTCG GCAGCACATATACTAAAAT-3' and reverse, 5'-CGCTTC ACGAATTTGCGTGTCAT-3'; miR-29a-3p, 5'-CGCTAGCAC CATCTGAAATCGGTTA-3'; glyceraldehyde-3-phosphate dehydrogenase (GAPDH) forward, 5'-CCTCTGCGCCCT TGAGCTAGGA-3' and reverse, 5'-CACAAGAAGATGCGG CCGTCTC-3'; SPARC forward, 5'-GCTCCCATTGGCGAG TTTG-3' and reverse, 5'-GATGTAGTCCAGGTGGAGCTT GTG-3'; E-cadherin forward, 5'-CACCGATGGTGAGGG TACACAG-3' and reverse, 5'-GGCTTCAGGAATACATGG ACAAAGA-3'; Vimentin forward, 5'-AAAGCGTGGCTG CCAAGAA-3' and reverse, 5'-ACCTGTCTCCGGTACTCG TTTGA-3'; U6 (human) forward, 5'-CTCGCTTCGGCA GCACA-3' and reverse, 5'-AACGCTTCACGAATTTGC GT-3'; miR-29a-3p (human) forward, 5'-TAGCACCATCTG AAATCGGTTA-3' and reverse, 5'-TGGTGTCGTGGAGTC G-3'; GAPDH (human) forward, 5'-GCACCGTCAAGGCTG AGAAC-3' and reverse, 5'-TGGTGAAGACGCCAGTGGA-3'; SPARC (human) forward, 5'-ACATAAGCCCAGTTCATC ACCA-3' and reverse, 5'-ACAACCGATTCACCAACTCCA-3'; E-cadherin (human) forward, 5'-GGATTGCAAATTCCTGCC ATTC-3' and reverse, 5'-AACGTTGTCCCGGGTGTCA-3'; and vimentin (human) forward, 5'-GGAAGGCGAGGAGAG CAGGATT-3' and reverse, 5'-TTCAAGGTCATCGTGATG CTGAGAAG-3'. qPCR thermocycling conditions were as follows: Denaturation at $95^{\circ} \mathrm{C}$ for $30 \mathrm{sec}$, annealing at $95^{\circ} \mathrm{C}$ for $5 \mathrm{sec}$, and extension at $60^{\circ} \mathrm{C}$ for $34 \mathrm{sec}$ for 40 cycles. The miRNA and mRNA levels were normalized to U6 or GAPDH, respectively. The fold-change for each gene was calculated using the $2^{-\Delta \Delta \mathrm{Cq}}$ method (13).

Western blotting. The lung tissues and cells were lysed in a mixture of RIPA lysis buffer (Beyotime Institute of Biotechnology) and protease inhibitors (Thermo Fisher Scientific, Inc.). The protein concentration of samples was measured using a BCA protein assay kit (Applygen Technologies, Inc.). Equal amounts of total protein $(20 \mu \mathrm{l})$ were loaded onto $10 \%$ sodium dodecyl sulfate-polyacrylamide gels and subjected to electrophoresis, and then separated proteins were electro-transferred onto PVDF membranes (Thermo Fisher Scientific, Inc.). Membranes were blocked with $5 \%$ skimmed milk or 5\% BSA (cat. no. A8020; Beijing Solarbio Science \& Technology Co., Ltd.) for $1 \mathrm{~h}$ and incubated at $4^{\circ} \mathrm{C}$ overnight with primary antibodies against the following proteins: SPARC (cat. no. 8725; 1:1,000; Cell Signaling Technology, Inc.), E-cadherin (cat. no. 3195; 1:1,000; Cell Signaling Technology, Inc.), vimentin (cat. no. 5741; 1:1,000; Cell Signaling Technology, Inc.), ERK (cat. no. 4695; 1:1,000; Cell Signaling Technology, Inc.), p-ERK (cat. no. 4370; 1:2,000; Cell Signaling Technology, Inc.) and GADPH (cat. no. 21612; 1:5,000; Signalway Antibody LLC). Following which, membranes were incubated at room temperature for $1 \mathrm{~h}$ with a secondary antibody (anti-rabbit IgG; cat. no. L3012; 1:5,000; Signalway Antibody LLC). An ECL kit (cat. no. BL520A; Biosharp Life Sciences) was used to view the protein bands, and ImageJ software version 1.8.0 (National Institutes of Health) was applied for the analysis of the relative intensities of protein bands.

Statistical analysis. Data were analyzed using GraphPad Prism 6 software (GraphPad Software, Inc.) and expressed as the mean $\pm \mathrm{SD}$ of three independent experimental repeats. The statistical significance was determined by an unpaired Student's t-test or one-way ANOVA with Tukey's post hoc test for multiple comparisons. For non-normally distributed data, the significance was determined using a Kruskal-Wallis test with a post hoc Dunn's multiple comparison test. $\mathrm{P}<0.05$ was considered to indicate a statistically significant difference.

\section{Results}

Establishment of the NA mouse model. The mouse model was established and presented with the following features of NA: Presence of bronchial hyperresponsiveness, the accumulation of inflammatory cells in the lung, particularly increased neutrophils, as well as a high number of neutrophils in the BALF. As shown in the H\&E staining images, NA mice exhibited a thick basement membrane and increased inflammatory cell infiltration around the bronchus (Fig. 1A). Airway resistance was elevated in the NA group when compared with the NC group after methacholine challenges at doses of 25 and $50 \mathrm{mg} / \mathrm{ml}$ (Fig. 1B). Compared with the NC mice, the total number of cells and neutrophils were significantly increased, while no eosinophilic cells were observed in the BALF of NA mice (Fig. 1C).

miR-29a-3p and SPARC are involved in NA. miR-29a-3p and SPARC expression levels were determined via RT-qPCR. Compared with the NC group, miR-29a-3p expression was decreased (Fig. 2A), while SPARC expression was increased in NA mice (Fig. 2B and E). The levels of EMT-related markers in the lung of NA mice were then determined. The results showed that the mRNA and protein expression levels of the epithelial marker, E-cadherin, were decreased in NA mice compared with the NC group (Fig. 2C and E). On the contrary, the mRNA and protein expression levels of the mesenchymal marker, vimentin, were increased in NA mice compared with the NC group (Fig. 2D and E). The data revealed that the NA mouse model exhibited an EMT phenotype with decreased levels of miR-29a-3p and increased SPARC.

SPARC regulates EMT in $16 \mathrm{HBE}$ cells. Next, the function of SPARC during EMT was investigated in 16HBE cells. SPARC was overexpressed in $16 \mathrm{HBE}$ cells following LV-SPARC transfection (Fig. 3A). The mRNA expression of E-cadherin was significantly reduced in the LV-SPARC group compared with the $\mathrm{NeC}$ group, whereas mRNA expression of vimentin was significantly increased by LV-SPARC administration (Fig. 3B). E-cadherin protein expression was reduced, while vimentin expression was elevated in SPARC-treated $16 \mathrm{HBE}$ cells compared with the LV-control group, as analyzed via western blotting (Fig. 3C), suggesting that SPARC participated in the EMT of $16 \mathrm{HBE}$ cells. 
A

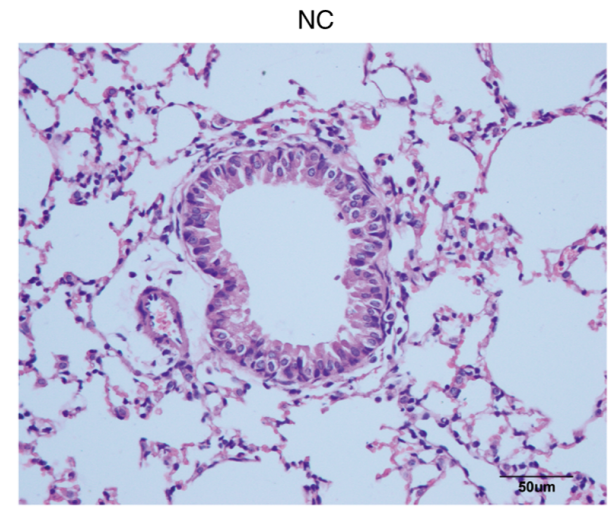

B

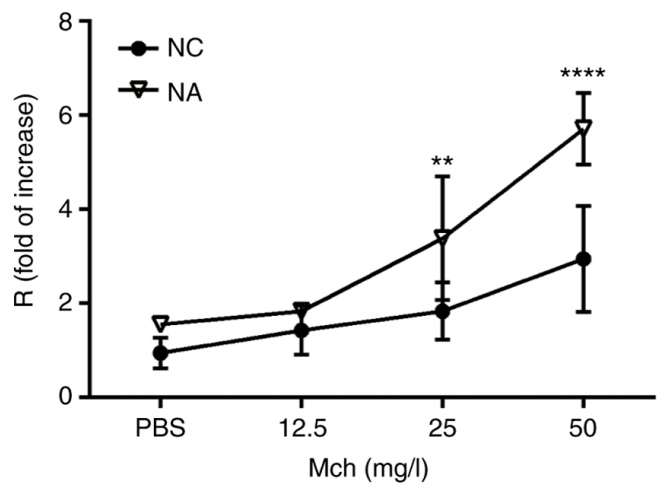

NA

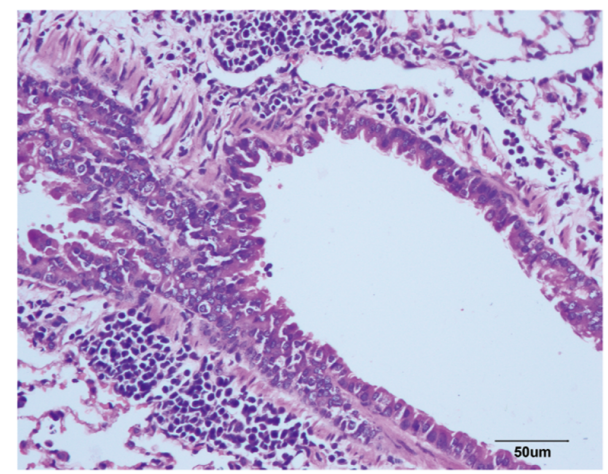

C

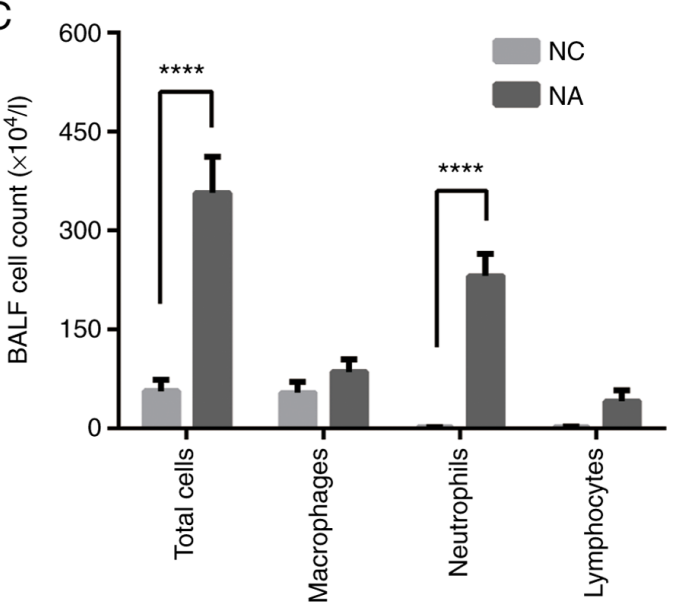

Figure 1. Establishment of the NA mouse model. (A) Representative images of hematoxylin and eosin-stained lung tissue (magnification, x100). (B) Airway resistance of the mouse model. (C) The differential cell counts in the BALF. $n=6$ mice per group. The results are presented as the mean \pm SD. Data were analyzed using a two-way ANOVA. ${ }^{* *} \mathrm{P}<0.01,{ }^{* * * * *} \mathrm{P}<0.0001$ vs. NC group. NC, normal control; NA, neutrophilic asthma; Mch, methacholine; BALF, bronchoalveolar fluid.

A
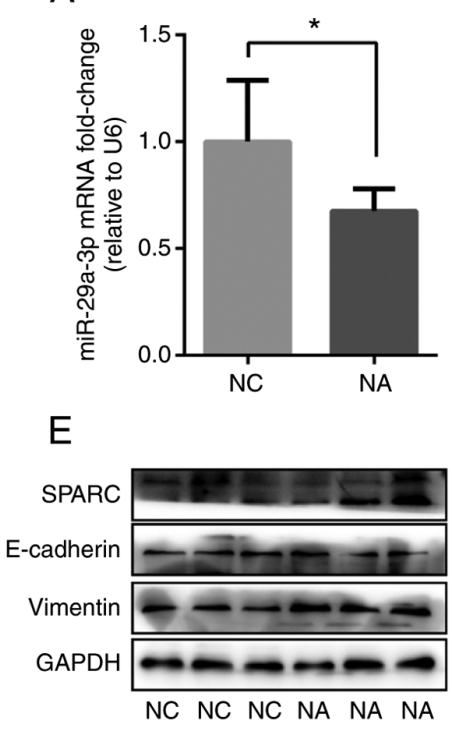

B
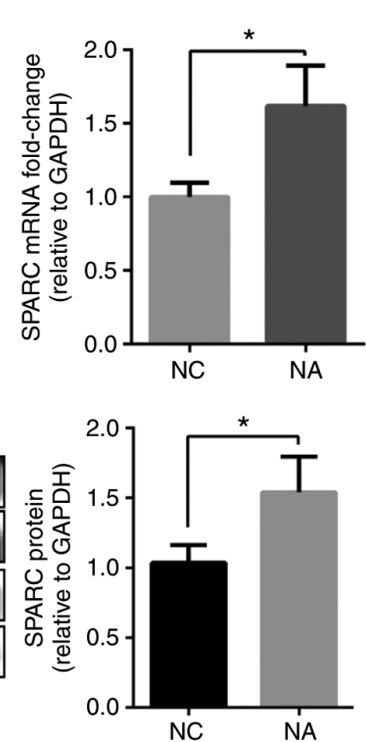

C
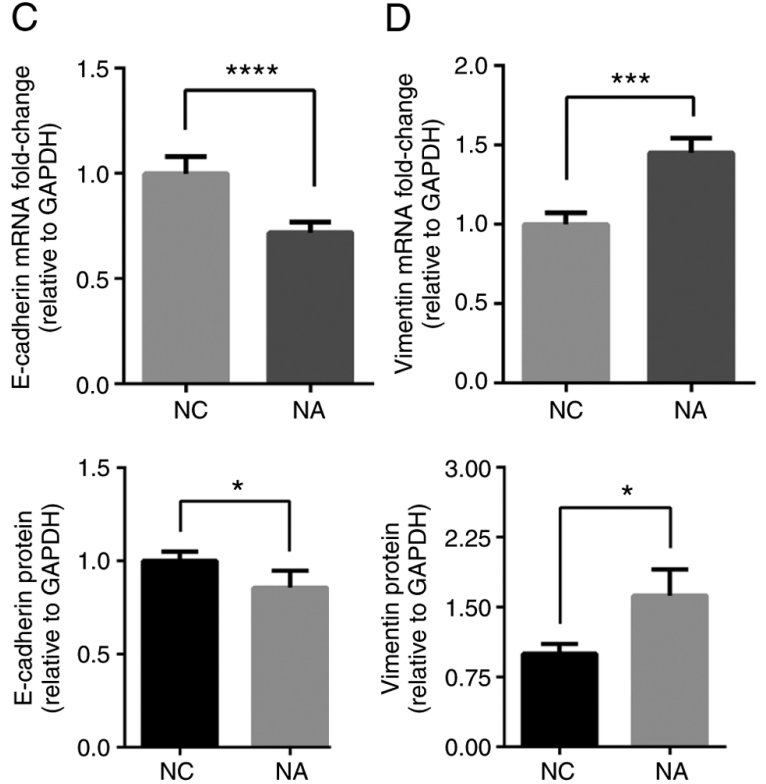

Figure 2. miR-29a-3p and SPARC are involved in NA. (A) miR-29a-3p, (B) SPARC, (C) E-cadherin and (D) Vimentin mRNA expression as measured via reverse transcription-quantitative PCR. (E) SPARC, E-cadherin and Vimentin protein expression levels were measured by western blotting. $\mathrm{n}=6 \mathrm{mice}$ per group. The results are presented as the mean $\pm \mathrm{SD}$. Data were analyzed using an unpaired Student's t-test. ${ }^{*} \mathrm{P}<0.05,{ }^{* * *} \mathrm{P}<0.001,{ }^{* * * *} \mathrm{P}<0.0001$. NC, normal control; $\mathrm{NA}$, neutrophilic asthma; miR, microRNA; SPARC, secreted protein acidic rich in cysteine.

miR-29a-3p regulates SPARC expression in vitro. miRNAs are hypothesized to be involved in various diseases where they inhibit gene expression through binding to the 3'UTR of the target gene (6). In the present study, the target gene of 
A

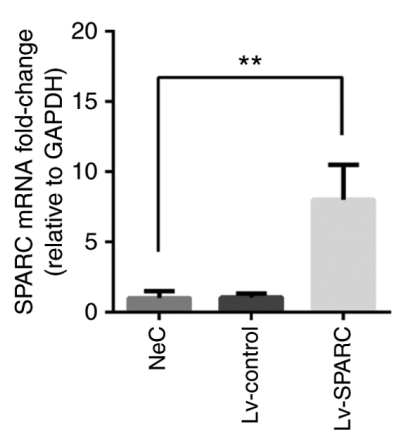

C

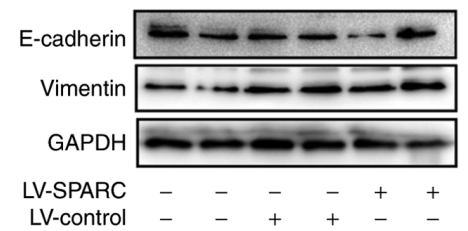

B
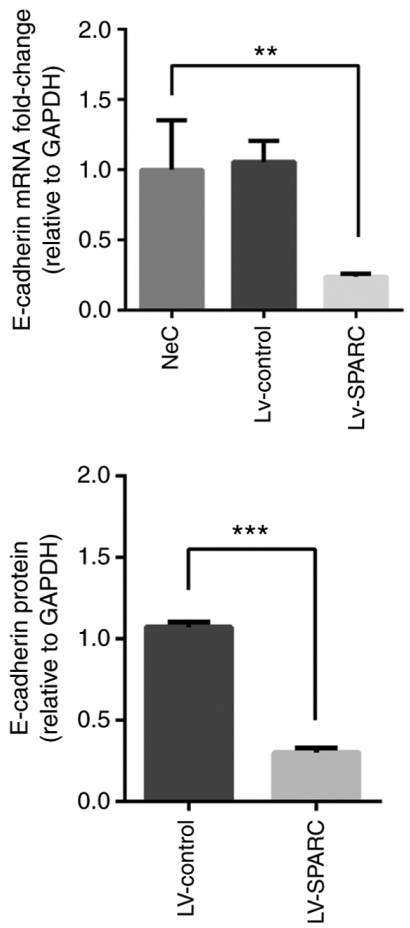
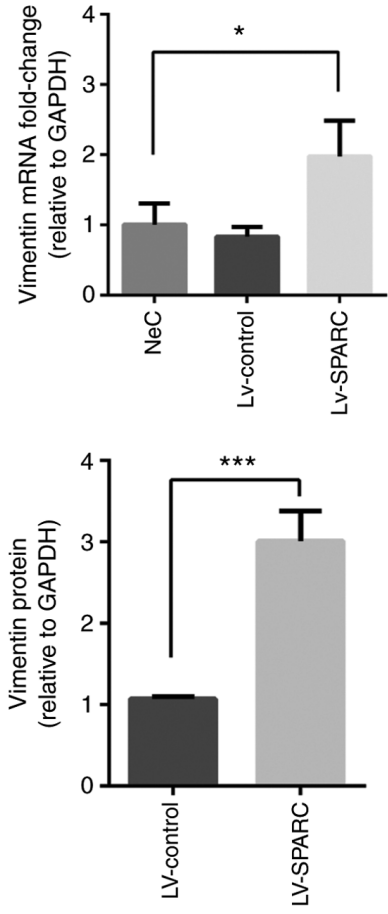

Figure 3. Effects of SPARC on the epithelial-mesenchymal transition of 16HBE cells. (A) SPARC mRNA was measured via RT-qPCR in 16HBE cells treated with LV-SPARC for 24 h. (B) mRNA expression levels of E-cadherin and Vimentin were measured via RT-qPCR. (C) Protein expression levels of E-cadherin and Vimentin were measured by western blotting. Three independent experiments were conducted for each treatment. The data are expressed as the mean \pm SD. Data were analyzed using a one-way ANOVA. ${ }^{*} \mathrm{P}<0.05,{ }^{* *} \mathrm{P}<0.01,{ }^{* * *} \mathrm{P}<0.001 . \mathrm{LV}$, lentivirus; SPARC, secreted protein acidic rich in cysteine; RT-qPCR, reverse transcription-quantitative PCR; NeC, negative control group.

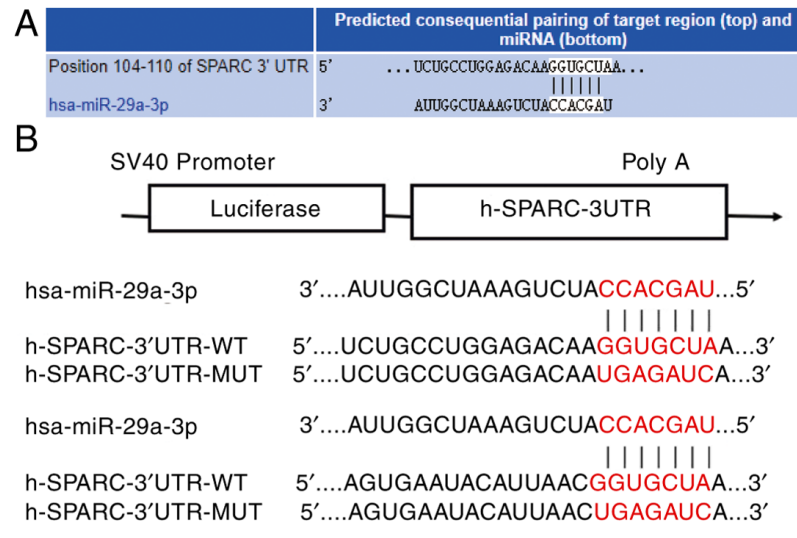

\begin{tabular}{|c|c|c|c|c|c|}
\hline $\begin{array}{c}\text { Site } \\
\text { type }\end{array}$ & $\begin{array}{c}\text { Context++ } \\
\text { score }\end{array}$ & $\begin{array}{c}\text { Context++ score } \\
\text { percentile }\end{array}$ & $\begin{array}{c}\text { Weighted } \\
\text { context++ score }\end{array}$ & $\begin{array}{c}\text { Conserved branch } \\
\text { length }\end{array}$ & P CT \\
\hline $\begin{array}{c}\text { 7mer- } \\
\text { A1 }\end{array}$ & -0.32 & 94 & -0.32 & 10.144 & 0.95 \\
\hline
\end{tabular}
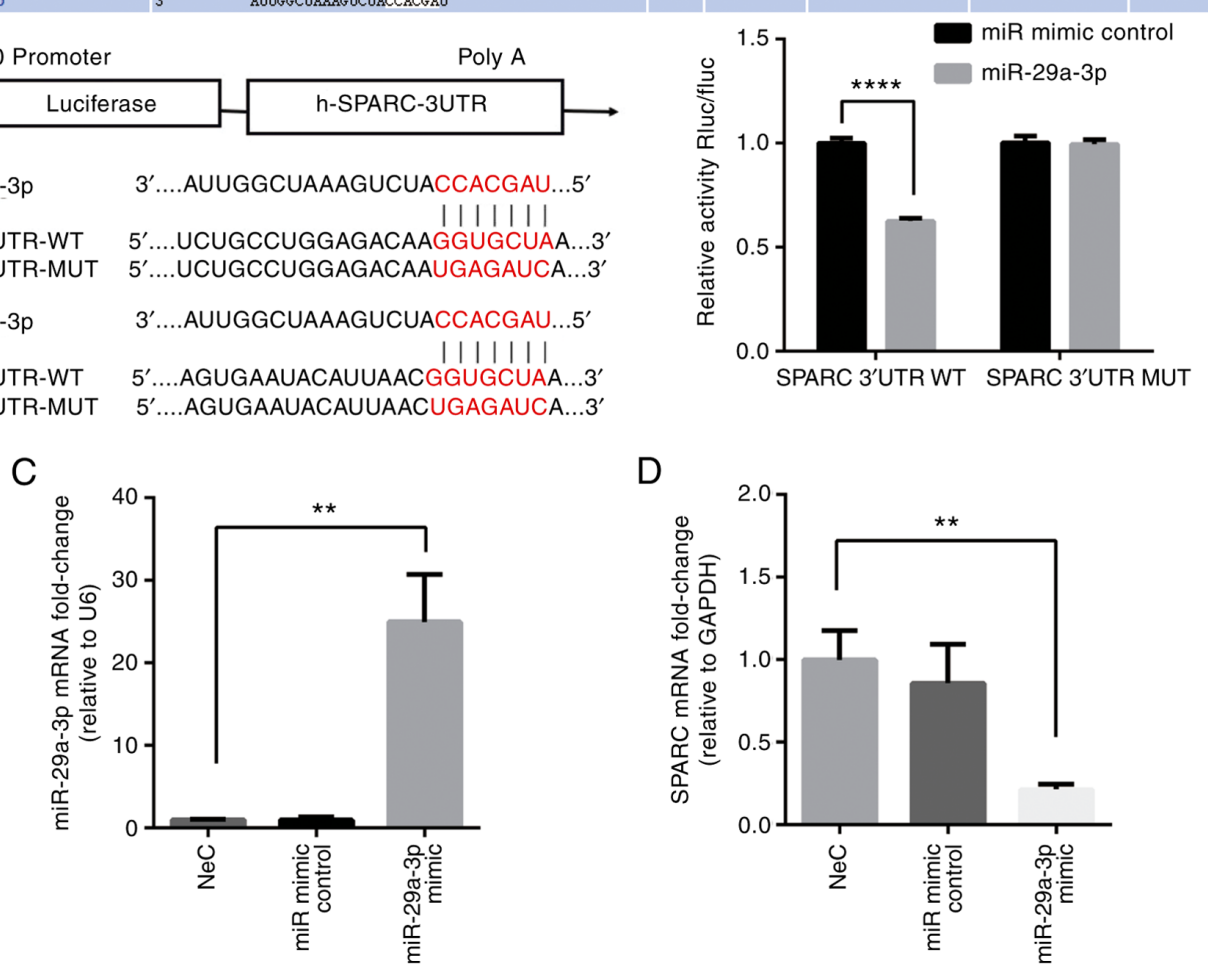

Figure 4. SPARC is the target gene for miR-29a-3p. (A) The predicted miR-29a-3p binding sites in the SPARC 3 'UTR. (B) The direct regulation of miR-29a-3p to SPARC was determined using dual-luciferase reporter gene assays. (C) miR-29a-3p and (D) SPARC mRNA expression as measured via reverse transcription-quantitative PCR in miR-29a-3p mimic-treated 16HBE cells. Three independent experiments were conducted for each treatment. The data are expressed as the mean $\pm \mathrm{SD}$. Data were analyzed using one-way or two-way ANONA as applicable. ${ }^{* *} \mathrm{P}<0.01,{ }^{* * * * *} \mathrm{P}<0.0001$. UTR, untranslated region; WT, wild-type; MUT, mutant; SPARC, secreted protein acidic rich in cysteine; miR, microRNA; NeC, negative control group. 
A

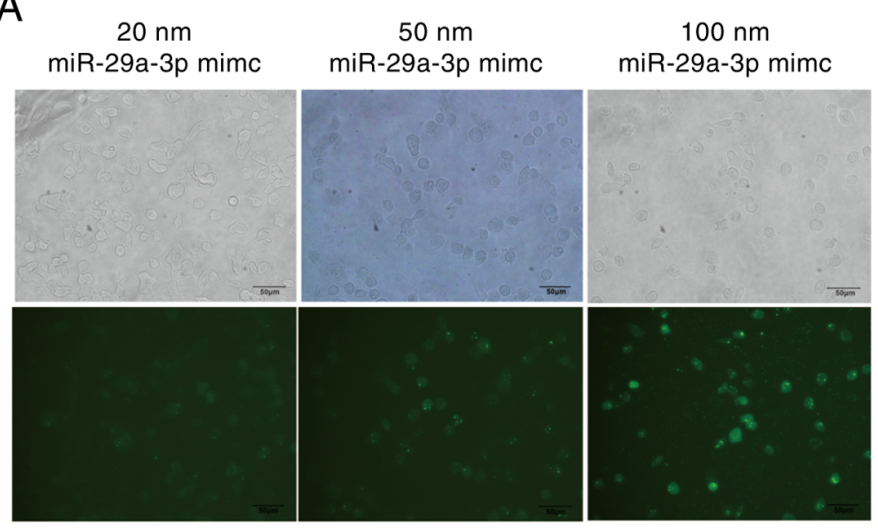

C

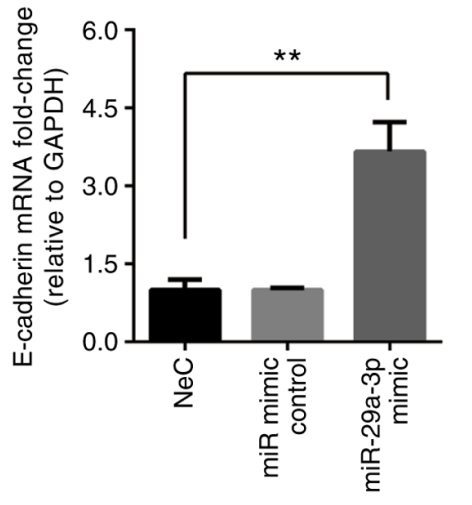

E

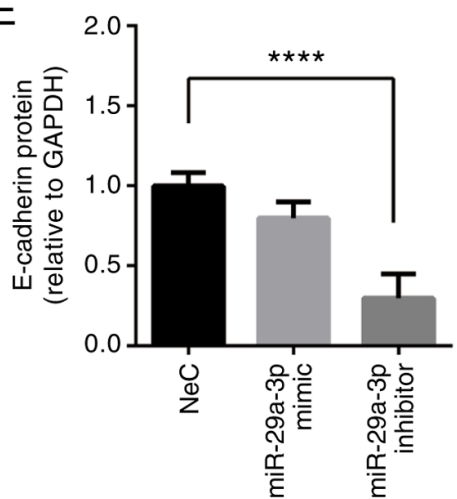

B
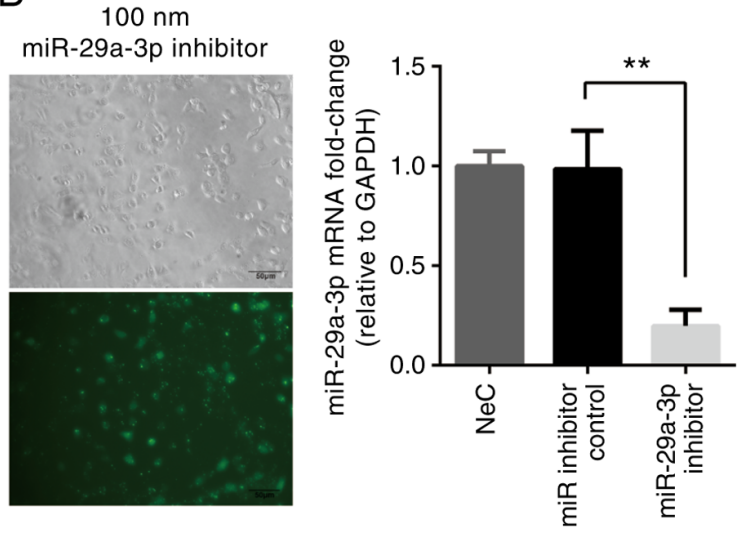

D
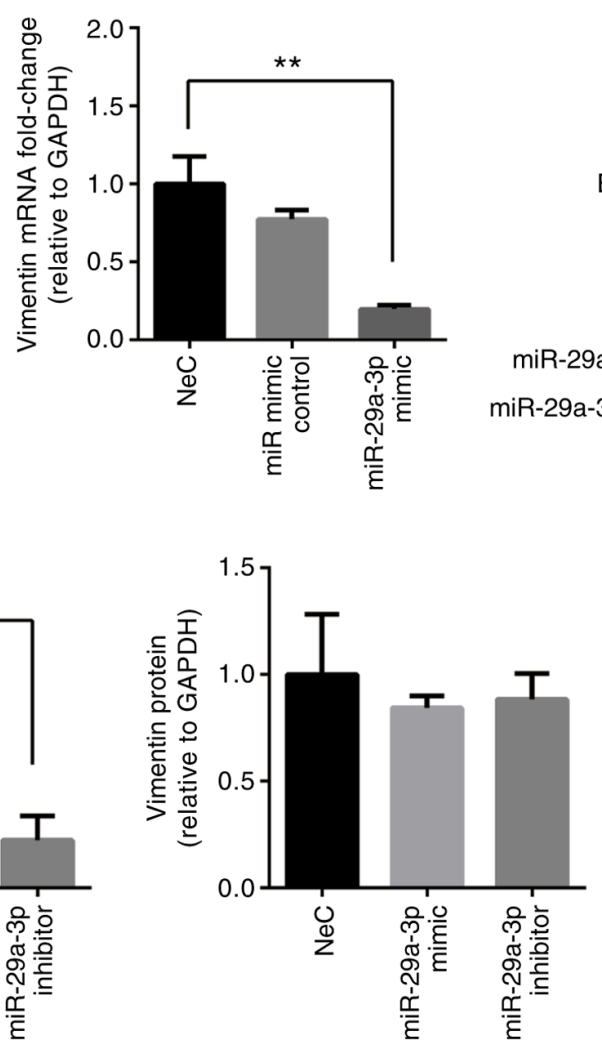

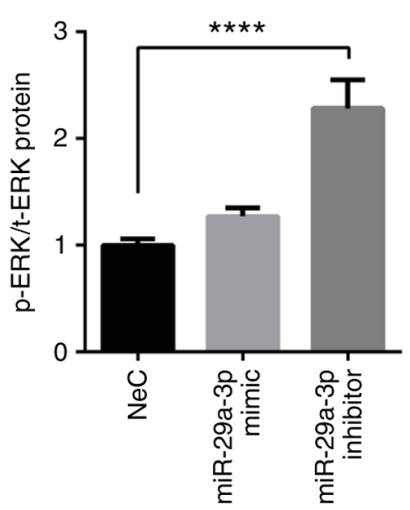

Figure 5. miR-29a-3p regulates the epithelial-mesenchymal transition of 16HBE cells. (A) Transfection efficiency was evaluated via fluorescence microscopy. (B) miR-29a-3p inhibitor induced the knockdown of miR-29a-3p. (C) E-cadherin and vimentin expression was measured via reverse transcription-quantitative PCR. (D and E) E-cadherin, Vimentin and p-ERK protein expression levels were measured via western blotting. Three independent experiments were conducted for each treatment. The data are expressed as the mean \pm SD. Data were analyzed using one-way ANOVA. ${ }^{* *} \mathrm{P}<0.01,{ }^{* * * * *} \mathrm{P}<0.0001$. miR, microRNA; $\mathrm{p}-$, phosphorylated; t-, total protein; $\mathrm{NeC}$, negative control group.

miR-29a-3p was predicted using the online bioinformatics software, TargetScan. The results showed that SPARC was a target gene for miR-29a-3p and the predicted binding site is shown in Fig. 4A. Dual-luciferase reporter gene assays demonstrated the direct regulation of miR-29a-3p by SPARC (Fig. 4B). Furthermore, the expression of SPARC in cultured 16HBE cells treated with a miR-29a-3p mimic was determined. miR-29a-3p expression following transfection is shown in Fig. 4C. It was found that SPARC mRNA expression was reduced after transfection of $16 \mathrm{HBE}$ cells with a miR-29a-3p mimic compared with the $\mathrm{NeC}$ group (Fig. 4D). These data supported the concept that reducing the expression of miR-29a-3p can regulate the mRNA synthesis of SPARC.
Effect of miR-29a-3p on EMT in 16HBE cells. Transfection efficacy was higher in $16 \mathrm{HBE}$ cells administrated with miR-29a-3p mimic at $100 \mathrm{nM}$, rather than at 20 or $50 \mathrm{nM}$, as determined using fluorescence microscopy (Fig. 5A). Transfection of $16 \mathrm{HBE}$ cells with a miR-29a-3p inhibitor was shown to successfully induce the knockdown of miR-29a-3p compared with the miR inhibitor control group (Fig. 5B). E-cadherin expression was increased, while vimentin expression was decreased in 16HBE cells transfected with the miR-29a-3p mimic at $100 \mathrm{nM}$ compared with the $\mathrm{NeC}$ group, as measured via RT-qPCR (Fig. 5C). E-cadherin expression was decreased, while p-ERK was increased in the miR-29a-3p inhibitor group compared with the $\mathrm{NeC}$ group, which was 

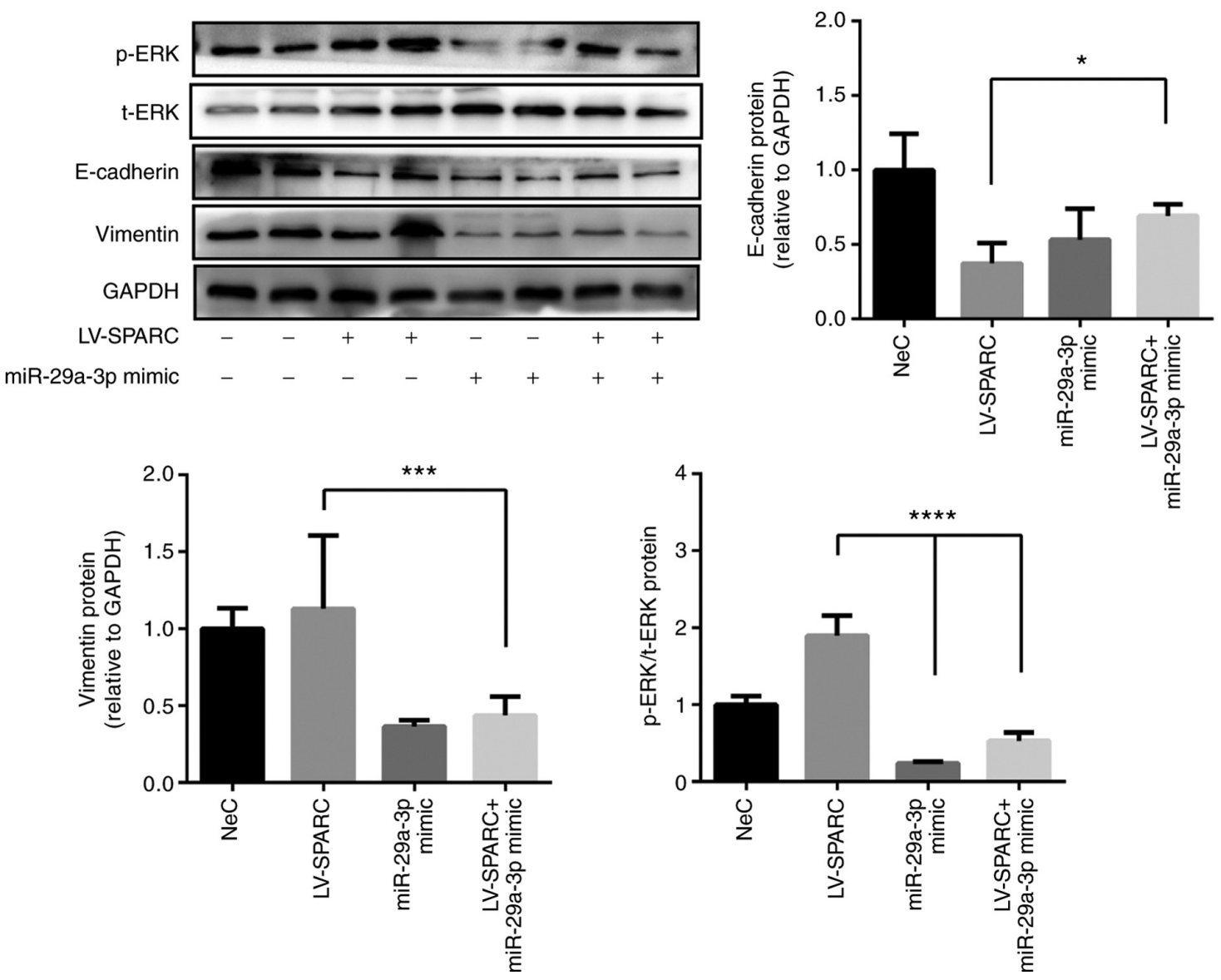

Figure 6. miR-29a-3p suppresses the epithelial-mesenchymal transition of SPARC-induced 16HBE cells. E-cadherin, Vimentin and p-ERK protein expression levels were assessed by western blotting. Representative images and semi-quantification of protein expression are shown. Three independent experiments were conducted for each treatment. The data are expressed as the mean \pm SD. Data were analyzed using one-way ANOVA. " $\mathrm{P}<0.05,{ }^{* * * *} \mathrm{P}<0.001,{ }^{* * * * *} \mathrm{P}<0.0001$. miR, microRNA; SPARC, secreted protein acidic rich in cysteine; p-, phosphorylated; t-, total protein; LV, lentivirus; NeC, negative control group.

verified via western blotting (Fig. 5D and E). Vimentin protein was not affected by administration of the miR-29a-3p inhibitor. These data indicated that miR-29a-3p regulated the mRNA expression of EMT-related markers, while the effect on protein expression was not significant. We speculated that a post-translational modification may be involved in the protein expression process. p-ERK expression increased after inhibitor administration, implying that ERK may participate in the process of EMT formation.

miR-29a-3p reduces SPARC-induced EMT in 16HBE cells. Transfection with the miR-29a-3p mimic increased E-cadherin expression, and decreased vimentin expression in 16HBE cells pretreated with LV-SPARC compared with transfection with LV-SPARC alone, as determined via western blotting (Fig. 6). This suggested that miR-29a-3p administration reversed the SPARC-induced EMT observed in 16HBE cells. p-ERK expression was upregulated by transfection with LV-SPARC alone, but this was reversed by administration of the miR-29a-3p mimic (Fig. 6). These results implied that ERK may be involved in the EMT process and that it is regulated by miR-29a-3p.

\section{Discussion}

It is commonly known that patients with NA may exhibit irreversible airflow obstruction and poor response to treatment $(3,4)$. Dysregulated extracellular matrix (ECM) remodeling has also been reported to result in EMT, which is considered to be involved in airway remodeling, which is the main cause of fixed airflow limitations that occur during asthma attacks (8). A previous study demonstrated that the loss of miR-29a-3p is crucial for ECM protein deposit and pulmonary fibrosis (14). However, findings concerning the underlying function of miR-29a-3p in asthma are still limited. The present study reported that miR-29a-3p was significantly decreased in the lung of the mouse model with NA and may mediate EMT via SPARC/ERK signaling pathway.

miR-29a-3p has been reported in a multitude of diseases, ranging from intrauterine inflammation in extremely preterm infants (15) and airway epithelial remodeling in chronic obstructive pulmonary disease (16) to proliferation and differentiation in retinal progenitors (17). Studies have reported that miR-29a-3p could be a therapeutic tool for certain diseases, such as pulmonary fibrosis (14) and Alzheimer's disease (18). The existing data revealed that miR-29a-3p inhibited Th17 cell differentiation and activation in a disease model with inflammatory bowel syndrome (19). Our previous study showed that NA was characterized by airway neutrophil inflammation and Th17 cell dominance (11).

In the present study, it was that miR-29a-3p was downregulated in NA when compared with the control mice. miR-29a-3p is well-characterized by its ability to regulate ECM proteins, 
including collagen, elastin and fibrillin, which play important roles in airway remodeling (20). For instance, downregulation of miR-29a-3p was found to result in the enhanced expression of the collagen proteins in pulmonary fibrosis (21). Moreover, it could attenuate TGF- $\beta 1$-induced fibrosis in primary human endometrial stromal cells (22) and mediate the remodeling process of airway epithelial cells (16). Therefore, we suggest that miR-29a-3p has a possible role in airway remodeling during NA. EMT is one of the mechanisms of airway remodeling in asthma (23). In the present study, it was found that the NA mouse model exhibited the EMT phenotype with decreased miR-29a-3p levels, suggesting it may be involved in airway remodeling of NA. It was demonstrated that miR-29a-3p regulated EMT in cultured 16HBE cells, which was similar to the regulation of miR-29a-3p in fibroblast accumulation in the kidneys (24). However, the present study also demonstrated that while E-cadherin and vimentin mRNA expression changed significantly, protein expression did not show the same obvious alternations. We speculated that this phenomenon may be related to post-translational modification. This deduction needs further research and discussion, which is a limitation of this study.

miRNAs regulate gene expression by binding to the 3'UTR of the target genes (25). An increasing number of studies have demonstrated that miR-29a-3p exhibits negative regulation of mRNAs encoding ECM proteins that play essential roles in matrix deposition and EMT $(21,22)$. In the present study, the online bioinformatics software, TargetScan, was used to predict the target genes for miR-29a-3p and SPARC. SPARC is a molecule that regulates cell proliferation, differentiation, ECM deposition and EMT, as well as participating in airway remodeling of chronic airways disease (26). Studies have shown that SPARC-null mice exhibited reduced collagen deposition and tissue fibrosis $(27,28)$. SPARC inhibition may, therefore, represent a potential therapeutic approach in fibrotic diseases (29). The present data showed that SPARC was upregulated in the lung of the mouse model with NA, implying that it may participate in airway remodeling. SPARC was reported to participate in the EMT of cancer cells $(30,31)$. In the current study, it was found that SPARC overexpression increased vimentin and decreased E-cadherin expression in vitro, suggesting that this molecule may contribute to EMT of $16 \mathrm{HBE}$ cells.

The direct link between miR-29a-3p and SPARC was verified using dual-luciferase reporter gene assays. Moreover, it was found that miR-29a-3p administration reversed the SPARC-induced EMT formation in 16HEB cells, supporting the concept that this miRNA inhibited EMT by suppressing SPARC synthesis directly. The data implied that the regulation of miR-29a-3p may participate in EMT of a mouse model with NA. The regulation of miR-29a-3p by SPARC was not verified in vivo, due to restricted access to the animal center due to COVID-19, and this is a limitation of the current study.

The potential signaling pathways involved in the miR-29a-3p-mediated effects on the EMT of 16HBE cells were also studied in the present study. The function of SPARC has been reported to be mediated by the phosphorylation of c-Jun N-terminal kinase and p38-MAPK signaling pathways on limbal epithelial stem cells (32). SPARC is upregulated during the EMT process in lung cancer cells and overexpression of SPARC can induce the increased expression of
p-Akt and p-ERK (31). Consistent with the existing studies, the present study found that SPARC overexpression led to the elevated expression of p-ERK and miR-29a-3p inhibited this increase. This suggested that ERK may participate in the SPARC-induced EMT process mediated by miR-29a-3p and SPARC observed in NA. Presenting the ratio of p-protein/total protein may improve the evidence for the activation of this signaling pathway. The band for total protein expression of ERK was not present because of the experimental design and the restricted laboratory access due to COVID-19, which was a limitation of this study.

In conclusion, the current study found that miR-29a-3p expression was decreased, while SPARC was elevated in a mouse model of NA. SPARC was observed to induce EMT in cultured $16 \mathrm{HBE}$ cells in vitro and this was directly targeted by miR-29a-3p, and may be mediated by p-ERK. The data suggested that miR-29a-3p may participate in the airway remodeling of NA.

\section{Acknowledgements}

The authors would like to thank Dr Dev Sooranna of Imperial College London (London, UK) for editing the manuscript.

\section{Funding}

This study was supported by International Communication of Guangxi Medical University Graduate Education, Guangxi Natural Science Foundation (grant no.2018GXNSFAA281256), Innovation Project of Guangxi Graduate Education (grant no. YCBZ2019045), Chen Xiaoping Foundation For The Development of Science and Technology of Hubei Province (grant no. CXPJJH1 1900003-17), Guangxi Health and Health Committee Project (grant no. Z20190762).

\section{Availability of data and materials}

The datasets used and/or analyzed during the current study are available from the corresponding author on reasonable request.

\section{Authors' contributions}

$\mathrm{XZ}$ conceived the present study, performed the experiments, analyzed the data, and wrote the manuscript. JX and HS conducted the animal studies. QW analyzed and interpreted the data. GN designed and supervised the study, critically revised the manuscript for intellectual content, and was primarily responsible for the writing of the manuscript. XZ and JX confirm the authenticity of all the raw data. All authors have read and approved the final manuscript.

\section{Ethics approval and consent to participate}

The present study was approved by The Ethics Committee of The First Affiliated Hospital of Guangxi Medical University [approval no. 2019 (KY-E-035); Nanning, China].

\section{Patient consent for publication}

Not applicable. 


\section{Competing interests}

The authors declare that they have no competing interests.

\section{References}

1. Szefler SJ, Fitzgerald DA, Adachi Y, Doull IJ, Fischer GB Fletcher M, Hong J, García-Marcos L, Pedersen S, Østrem A, et al: A worldwide charter for all children with asthma. Pediatr Pulmonol 55: 1282-1292, 2020.

2. Seys SF, Lokwani R, Simpson JL and Bullens DMA: New insights in neutrophilic asthma. Curr Opin Pulm Med 25: 113-120, 2019.

3. Gibson PG and Foster PS: Neutrophilic asthma: Welcome back! Eur Respir J 54: 1901846, 2019.

4. Postma DS and Rabe KF: The asthma-COPD overlap syndrome. N Engl J Med 373: 1241-1249, 2015.

5. Jonas $\mathrm{S}$ and Izaurralde E: Towards a molecular understanding of microRNA-mediated gene silencing. Nat Rev Genet 16: 421-433, 2015.

6. Kwon JJ, Factora TD, Dey S and Kota J: A systematic review of miR-29 in cancer. Mol Ther Oncolytics 12: 173-194, 2018.

7. Gao Y, Qiao H, Lu Z and Hou Y: miR-29 promotes the proliferation of cultured rat neural stem/progenitor cells via the PTEN/AKT signaling pathway. Mol Med Rep 20: 2111-2118, 2019.

8. Sohal SS, Ward C and Walters EH: Importance of epithelial mesenchymal transition (EMT) in COPD and asthma. Thorax 69: $768,2014$.

9. Haddad A, Gaudet M, Plesa M, Allakhverdi Z, Mogas AK, Audusseau S, Baglole CJ, Eidelman DH, Olivenstein R, Ludwig MS and Hamid Q: Neutrophils from severe asthmatic patients induce epithelial to mesenchymal transition in healthy bronchial epithelial cells. Respir Res 20: 234, 2019.

10. Wilson RH, Whitehead GS, Nakano H, Free ME, Kolls JK and Cook DN: Allergic sensitization through the airway primes Th17-dependent neutrophilia and airway hyperresponsiveness. Am J Respir Crit Care Med 180: 720-730, 2009.

11. Zhang X, Zhang M, Jiang M and Nong G: Effect of IL-7 on Th17 cell responses in a mouse model of neutrophilic asthma. Mol Med Rep 22: 1205-1112, 2020.

12. Hou C, Kong J, Liang Y, Huang H, Wen H, Zheng X, Wu L and Chen Y: HMGB1 contributes to allergen-induced airway remodeling in a murine model of chronic asthma by modulating airway inflammation and activating lung fibroblasts. Cell Mol Immunol 12: 409-423, 2015.

13. Livak KJ and Schmittgen TD: Analysis of relative gene expression data using real-time quantitative PCR and the 2(-Delta Delta C(T)) method. Methods 25: 402-408, 2001

14. Xiao J, Meng XM, Huang XR, Chung AC, Feng YL, Hui DS, Yu CM, Sung JJ and Lan HY: miR-29 inhibits bleomycin-induced pulmonary fibrosis in mice. Mol Ther 20: 1251-1260, 2012.

15. Pavlek LR, Vudatala S, Bartlet CW, Buhimschi IA, Buhimschi CS and Rogers LK: miR-29b is associated with perinatal inflammation in extremely preterm infants. Pediatr Res 89: 889-893, 2021.

16. Chi Y, Di Q, Han G, Li M and Sun B: Mir-29b mediates the regulation of Nrf2 on airway epithelial remodeling and Th1/Th2 differentiation in COPD rats. Saudi J Biol Sci 26: 1915-1921, 2019.

17. Zhang Y, Shen B, Zhang D, Wang Y, Tang Z, Ni N, Jin X, Luo M, Sun H and Gu P: miR-29a regulates the proliferation and differentiation of retinal progenitors by targeting Rbm8a. Oncotarget 8: 31993-32008, 2017.
18. Pereira PA, Tomás JF, Queiroz JA, Figueiras AR and Sousa F: Recombinant pre-miR-29b for Alzheimer s disease therapeutics. Sci Rep 6: 19946, 2016.

19. Fukata T, Mizushima T, Nishimura J, Okuzaki D, Wu X, Hirose H, Yokoyama Y, Kubota Y, Nagata K, Tsujimura N, et al: The supercarbonate apatite-MicroRNA complex inhibits dextran sodium sulfate-induced colitis. Mol Ther Nucleic Acids 12: 658-671, 2018.

20. He Y, Huang C, Lin X and Li J: MicroRNA-29 family, a crucial therapeutic target for fibrosis diseases. Biochimie 95: 1355-1359, 2013.

21. Cushing L, Kuang P and Lü J: The role of miR-29 in pulmonary fibrosis. Biochem Cell Biol 93: 109-118, 2015.

22. Li J, Cen B, Chen S and He Y: MicroRNA-29b inhibits TGF- $\beta 1$-induced fibrosis via regulation of the TGF- $\beta 1 / \mathrm{Smad}$ pathway in primary human endometrial stromal cells. Mol Med Rep 13: 4229-4237, 2016.

23. Pain M, Bermudez O, Lacoste P, Royer PJ, Botturi K, Tissot A, Brouard S, Eickelberg O and Magnan A: Tissue remodelling in chronic bronchial diseases: From the epithelial to mesenchymal phenotype. Eur Respir Rev 23: 118-130, 2014.

24. Srivastava SP, Hedayat AF, Kanasaki $\mathrm{K}$ and Goodwin JE: microRNA crosstalk influences epithelial-to-mesenchymal, endothelial-to-mesenchymal, and macrophage-to-mesenchymal transitions in the kidney. Front Pharmacol 10: 904, 2019.

25. Krol J, Loedige I and Filipowicz W: The widespread regulation of microRNA biogenesis, function and decay. Nat Rev Genet 11: 597-610, 2010.

26. Wong SL and Sukkar MB: The SPARC protein: An overview of its role in lung cancer and pulmonary fibrosis and its potential role in chronic airways disease. Br J Pharmacol 174: 3-14, 2017.

27. Trombetta-Esilva J and Bradshaw AD: The function of SPARC as a mediator of fibrosis. Open Rheumatol J 6: 146-155, 2012

28. Bradshaw AD: The role of secreted protein acidic and rich in cysteine (SPARC) in cardiac repair and fibrosis: Does expression of SPARC by macrophages influence outcomes? J Mol Cell Cardiol 93: 156-161, 2016.

29. Wang JC, Lai S, Guo X, Zhang X, de Crombrugghe B, Sonnylal S, Arnett FC and Zhou X: Attenuation of fibrosis in vitro and in vivo with SPARC siRNA. Arthritis Res Ther 12: R60, 2010.

30. Zhang F, Zhang Y, Da J, Jia Z, Wu H and Gu K: Downregulation of SPARC expression decreases cell migration and invasion involving epithelial-mesenchymal transition through the p-FAK/p-ERK pathway in esophageal squamous cell carcinoma. J Cancer 11: 414-420, 2020.

31. Sun W, Feng J, Yi Q, Xu X, Chen Y and Tang L: SPARC acts as a mediator of TGF- $\beta 1$ in promoting epithelial-to-mesenchymal transition in A549 and H1299 lung cancer cells. Biofactors 44: 453-464, 2018

32. Zhu J, Wang LY, Li CY, Wu JY, Zhang YT, Pang KP, Wei Y, Du LQ, Liu M and Wu XY: SPARC promotes self-renewal of limbal epithelial stem cells and ocular surface restoration through JNK and p38-MAPK signaling pathways. Stem Cells 38: $134-145,2020$

This work is licensed under a Creative Common Attribution-NonCommercial-NoDerivatives 4.0 International (CC BY-NC-ND 4.0) License. 